\title{
Mixed integer non-linear programming and Artificial Neural Network based approach to ancillary services dispatch in competitive electricity markets
}

\author{
Bruno Canizes, João Soares, Pedro Faria, Zita Vale
}

\author{
A B S T R A C T
}

\begin{abstract}
Ancillary services represent a good business opportunity that must be considered by market players. This paper presents a new methodology for ancillary services market dispatch. The method considers the bids submitted to the market and includes a market clearing mechanism based on deterministic optimization.

An Artificial Neural Network is used for day-ahead prediction of Regulation Down, regulation-up, Spin Reserve and Non-Spin Reserve requirements.

Two test cases based on California Independent System Operator data concerning dispatch of Regulation Down, Regulation Up, Spin Reserve and Non-Spin Reserve services are included in this paper to illustrate the application of the proposed method: (1) dispatch considering simple bids; (2) dispatch considering complex bids.
\end{abstract}

Keywords:

Ancillary services

Artificial Neural Networks

Electricity markets

Linear programming

Mixed integer non-linear programming

Power systems

\section{Introduction}

Electric power industry is moving towards an increasing competition, with a variety of services previously provided by electric utilities being progressively unbundled [1-7]. Initially the attentions have mainly focused on the energy market but ancillary ser- vices (AS) are gaining more and more importance. Ancillary services have an essential role in the operation of electricity markets [8-12]. AS are generally defined as those services that are important to achieve targeted goals on power system security, reliable operation, frequency stability, as well as on voltage level and stability [13-15], in order to cover generation and transmission contingencies.

The definition of ancillary services prices and how those prices should change regarding the operating decisions is becoming a major issue due to the changes in the market structure $[9,16]$. The creation of a market for ancillary services aims at achieving mutual benefits for buyers and sellers. The Independent System Operator (ISO) aims at obtaining the necessary resources in order to maintain the system security at minimum cost, while providers look for the maximization of their profits from the sale of ancillary services.

Ancillary services are necessary, according to the Federal Energy Regulatory Commission (FERC), to support the power transmission from sellers to buyers, whereas the control areas and transmission 


\section{Nomenclature}

$N \quad$ total number of bids

$i \quad$ bid index $(i=1,2, \ldots, N)$

$k \quad$ ancillary service index ( 1 for Regulation Down, 2 for Regulation Up, 3 for spin, and 4 for non-spin)

$T \quad$ total number periods in hours

$t \quad$ period index $(t=1,2, \ldots, T)$

$Q_{k, t} \quad$ total capacity requirement for ancillary service $k$ in period $t$

Cmax $_{i, t}$ maximum capacity of bid $i$ in period $t$

$C_{k, i, t} \quad$ capacity of bid $i$ for ancillary service $k$ in period $t$

$X_{k, i, t} \quad$ accepted capacity of bid $i$ for ancillary service $k$ in period $t$

$P R_{k, i, t} \quad$ capacity reserve price of bid $i$ for ancillary service $k$ in period $t$
$P E_{k, i, t} \quad$ energy reserve price of bid $i$ for ancillary service $k$ in period $t$

$p \quad$ estimated probability of using reserve energy acquired for AS

$M m_{i} \quad$ minimum limit of remuneration for the whole $24 \mathrm{~h}$

$Y_{k, i, t} \quad$ decision variable of bid $i$ for service $k$ in period $t$

$Y l_{k, i, t} \quad$ minimum working hours of bid $i$ for service $k$ in each time period

Yup $_{k, i, t} \quad$ maximum working hours of bid $i$ for service $k$ in each time period

$M h_{i} \quad$ minimum consecutive working hours of bid $i$ utilities have the obligation to maintain a reliable operation of interconnected transmission system [17].

Regulation services - Regulation Up (RU) and Regulation Down (RD) - are the first level acting reserves which are updated around every $4 \mathrm{~s}$ depending on the system operator. The regulation keeps the continuous matching between load and generation and is controlled by Automatic Generation Control (AGC) systems [18,19].

The Spin Reserve (SR) is one of the most important AS required for maintaining power system reliability following a major contingency. In the same way, Non-Spin Reserve (NSR) has an important role in keeping the system security, since this reserve is used when SR cannot satisfy the reserve needs. Short-term prediction of dayahead reserve requirement helps the ISO making effective and timely decisions. Moreover, based on these forecasts, market participants can make decisions concerning their bidding strategies for day-ahead SR market $[18,19]$.

Two alternative methods are available for energy and ancillary service provision: simultaneous procurement and separate procurement [20], with advantages and drawbacks of each of these solutions. Because most ancillary services are provided by the same equipments that produce energy, the energy and AS markets are linked so any problem in one of these markets may cause a problem in the other [21].

In recent years, ancillary service markets design has become an important work area. Designing options of bid selection protocols and settlement rules for ancillary service markets are presented in [22]. An ancillary framework for managing power system security, based on a market for ancillary services, is presented in [23]. A detailed formulation of simultaneous energy and ancillary service auctions for integrated market systems by an optimal power flow is presented in [24]. A Security Constrained Unit Commitment model for energy and ancillary services auction, which can be used by an ISO to optimize reserve requirements in electricity markets, can be found in [25]. In [13], Vale et al. present an approach using linear programming and genetic algorithm approaches that has been used in MASCEM, a multi-agent electricity market simulator, to deal with ancillary services market simulation. An Artificial Neural Network approach for day-ahead RD, RU and NS forecasting which has been included in MASCEM [26] is described in [19]. [27] presents two mathematical models, the Security Constrained Unit Commitment (SCUC) and the Security Constrained Economic Dispatch (SCED) that are used to perform Unit Commitment and Economic Dispatch respectively in CAISO (California ISO) dayahead and Real Time Markets.

It is well known that the economic selling bids of energy that sellers present to the market operator can be simple or integrate complex conditions. The simple bids are economic offers of energy sale that sellers present for each time period and for each generation unit of which they own with a price and a quantity of energy.
In most of the cases the bids are not simple and integrate complex conditions of sale in order to achieve the producers (sellers) objectives and the needs of the ISO. The complex bids are those that comply with the requirements for the simple bids and also integrate any of the allowed conditions, such as: minimum remuneration for each bid, minimum working hours, and minimum consecutive working hours. Thus, in order to accommodate the objectives of the producers (sellers) and the needs of the ISO this paper proposes a methodology for AS market management and simulation. This methodology includes two distinct phases: the AS requirements forecasting and the AS dispatch. The AS requirements forecast based on Artificial Neural Networks (ANN) has been previously proposed by some of the authors of this paper [19]. The ANN is trained using AS historic data and are able to determine the amount of power to be guaranteed for each AS. The AS dispatch considers the bids submitted to the market and includes a market clearing mechanism based on linear programming and other considering complex bids based on mixed integer non-linear programming.

After this introduction section, this paper is organized as follows: Section 2 deals with ancillary services in electricity markets. Section 3 presents the methodology for the problem under study. Section 4 presents two case studies and the discussion of the obtained results. The presented case studies use real AS data from California Independent System Operator (CAISO) to forecast AS requirements. Finally, Section 5 presents the most relevant conclusions of the presented work.

\section{Ancillary services}

Generation must handle the load requirements; however, satisfying the demand is not the only requirement for power system operation. To ensure power system security and reliability, it is necessary to guarantee some extra energy generation, which can be seen as a set of additional services known as ancillary services (AS).

Generally, the Independent System Operator (ISO) has the responsibility of determining, getting and using the required AS.

In order to maintain the system in a secure operation state, the ISO must be able to [28]:

- control the frequency by keeping the load-demand balance;

- control the system voltage profile within certain bounds;

- maintain the system stability;

- prevent overloads in the transmission system;

- restore portions or entire system when and if required.

Besides this list, the system operator must also maintain the system integrity in the presence of unexpected events and contingencies. 
The ISO aims at minimizing AS costs, while encouraging the participants to provide the required AS. On the other hand, the energy producers can consider AS business opportunities in their strategic bidding in order to maximize their profits $[13,19]$.

Active power ancillary services - load/frequency control, including primary control, Automatic Generation Control (AGC), tertiary control, balancing service and black start provision - and reactive power AS - voltage/reactive power control - are included in ancillary services provision. The AS considered in this paper can be shortly defined as follows [13]:

- Regulation: generating units operating and synchronized with the ISO controlled grid. The produced active power can be incremented or decreased instantaneously through AGC. Regulation is used to maintain real-time balance in the system.

- Spin Reserves: are available almost instantaneously to stabilize the system frequency upon the outage of a large generation unit in the system. Their time response varies from a few seconds to about $5 \mathrm{~min}$ and require no notification.

- Non-Spin Reserves: generation resources are available but not running; the time frame varies from several minutes to half an hour or more. These reserves often require some manual intervention for their activation to be exercised quickly and reliably. Non-Spin Reserves are needed not only to stabilize the system frequency, but also to deal with the energy balance within a control area.

Ancillary services began to be defined in different electricity markets since the mid-90s. In the same period, the establishment of methodologies and tools for compensation or incomings has been initiated.

Electricity market liberalization made AS issues more complex as adequate procedures and methodologies are required to determine AS needs, the way they are obtained and priced in a competitive environment. As AS opportunities can be used by producers to increase their income, methods for portfolio optimization and reserve management [29] have been developed and tested.

\section{Proposed methodology}

The proposed methodology is for a day-ahead AS market and an Artificial Neural Network (ANN) based methodology [19] is used to forecast ancillary services (AS) requirements in the scope of AS market simulation. The forecasting of the following four ancillary services: Regulation Down, Regulation Up, Spin Reserve and NonSpin Reserve is addressed in this paper. This methodology can be easily integrated and coordinated with the real time balancing market. In the case of the California Independent System Operator (CAISO) the operative reserve requirements are calculated as $7 \%$ of load for each of Spin Reserve (SR) and Non-Spin Reserve (NSR), i.e., they depend on the load value. The demand forecast is updated in the trading day at every $30 \mathrm{~min}$. Thus, the proposed methodology can be coordinated and applied to the real time balancing market considering the new values of the load forecast and therefore the new requirements can be updated before the real time economic dispatch.

After processing the data, each ancillary service requirement is computed separately and the results are used as an input by the ancillary service market simulation. AS markets can use several clearing mechanisms (CM). The application of the chosen CM with the AS requirements and with the players bids produces the ancillary service dispatch as can be seen in Fig. 1.

The determination of the values of each AS requirement is an important task since the reliable operation of the system has to be guaranteed and, moreover, the cost of an additional unit of AS has not negligible costs. Therefore adequate tools for AS requirements forecast are needed.

The methodology which is presented is to be used by the market operator, but it is also useful for bid makers in order to simulate the behavior of the market clearing process and support their decision making. Thus, the proposed methodology is implemented in MASCEM [26] which is a multi-agent based electricity market simulator that uses sophisticated Artificial Intelligence based techniques to provide electricity market players with adequate decision-support. It uses a flexible and open approach that supports diversity of electricity market models and several tools to be used by market players and a balanced approach of technical and economic issues. Fig. 2 shows the architecture of MASCEM and where the proposed methodology is located. MASCEM can use the features and simulate a diversity of markets operators (CAISO, OMEL, NordPool, etc.) and can consider different time frames in each kind of market (day ahead market, real time balancing markets, etc.), for that it is only need define the respective time frames.

\subsection{ANN forecast}

The Artificial Neural Networks based method previously developed [19] is applied to ancillary services (AS) requirement forecast. Suitable study and knowledge about the problem in general, and the specific characteristics of the data to be processed are necessary.

For the forecast of the four AS (Regulation Down (RD), Regulation Up (RU), Spin Reserve (SR),and Non-Spin Reserve (NSR)) studied in this paper and used by the California Independent System Operator (CAISO), it was necessary to analyze the AS requirements. This forecast has been done in an isolated way by the authors in [19] and [30].

This forecast consists of three stages - training, test and validation stages. Each stage includes a feedback process that allows the Artificial Neural Network to learn from its mistakes and to correct its output by adjusting its internal weights. The training stage is of large importance since, for instance, the overtraining of neurons can seriously deteriorate the forecasting results. Furthermore, training the ANNs based on a training matrix, that is very different from the input matrix, can also make the forecasting useless [31].

In [32], CAISO historical data are studied, leading to the data presented in Table 1 . This historical data analysis is done for the SR service and the cross relation function between SR and the load was useful to decide the number of lagged values of load to be used to predict the SR requirements. In this paper, the same historical values are used for the four forecasted services.

Table 1 presents the input values of historical data used for the forecast of each service. Values of the Forecasted Demand (FD) and Required Service (RS) of the day $(n)$ that includes the hour $(h)$ to be forecasted and the eight days before are used. Generally, the values of FD and RS ( " $x$ " in Table 1) are used for the hour to be forecasted $(F)$ and in the previous days $(n-1, n-2, \ldots, n-8)$ as well as for the tree hours before the hour to be forecasted.

The work proposed in [32] uses an ANN with 24 neurons in the input layer and three neurons in the hidden layer. The work presented in this paper uses the ANN with the same input and structure, but with a different quantity of neurons in the hidden layer ( six neurons instead of 3 ). Both structures have one neuron in the output layer, which corresponds to the value to be forecasted. The neurons in the input layer and the neurons in the hidden layer are marked with " $x$ ", as can be seen in Table 1 .

The used ANN implementation uses Levenberg-Marquardt backpropagation function, updating weight and bias values according to Levenberg-Marquardt optimization [33]. This approach led to good results, with good performance regarding the computational time, although requiring more memory than other algo- 


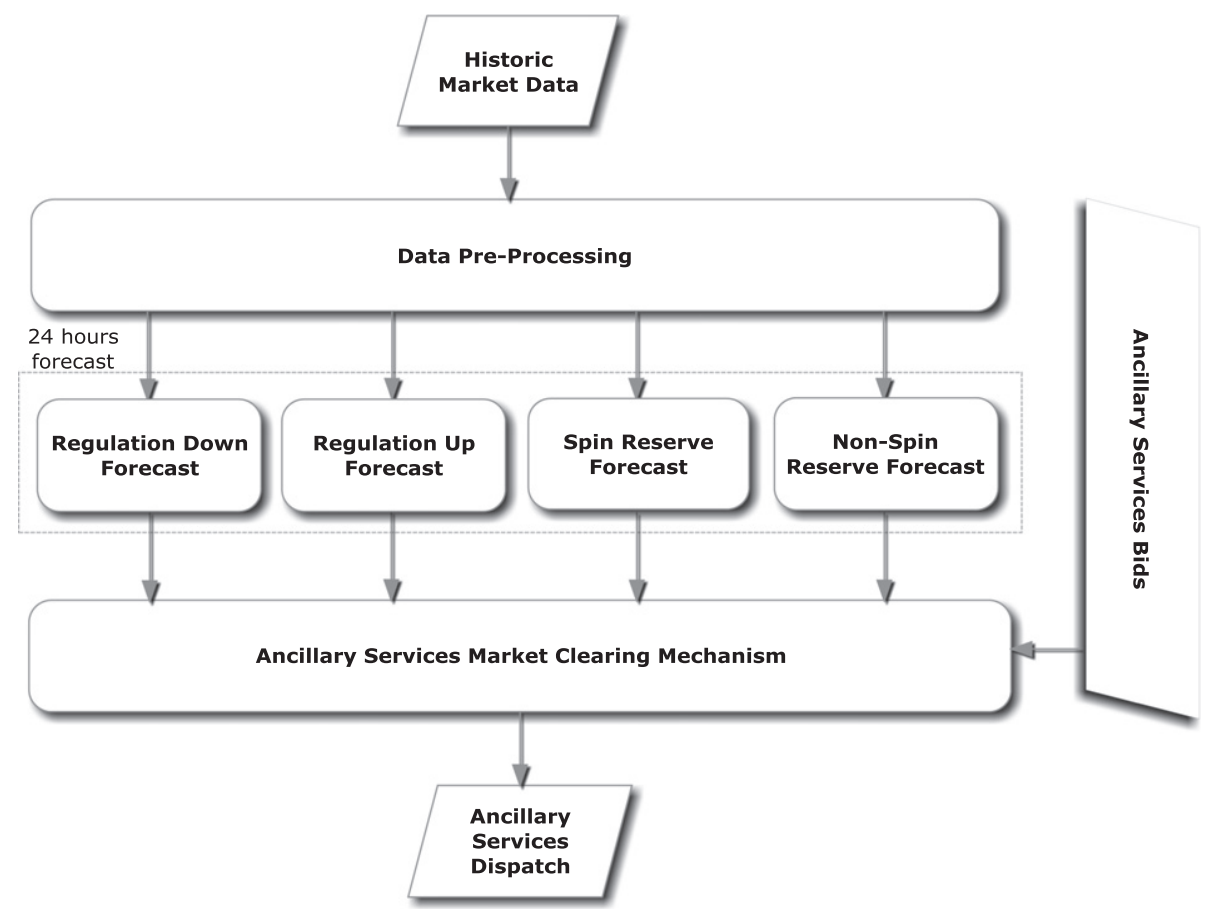

Fig. 1. Structure of ancillary services dispatch.

rithms. After the training, feed-forward backpropagation algorithm is applied to obtain the forecast.

The performance of the forecast model can be evaluated using the Mean Absolute Percentage Error (MAPE), which is determined with the forecasted values and the actually verified values. Smaller values of MAPE mean predicted values closer to the real ones. MAPE is calculated according to the following equation:

$M A P E=\frac{1}{24} \sum_{h=1}^{24} \frac{\left|R S_{h}^{\text {act }}-R S_{h}^{\text {for }}\right|}{R S_{h}^{\text {act }}} \times 100$

where $h$ is the hour for which the forecast is being undertaken, and $R S_{h}^{a c t}$ and $R S_{h}^{\text {for }}$ are the actual RS and the forecasted values, respectively.

\subsection{Ancillary services market clearing}

Generators can provide power up to their maximum capacity. This capacity can be split into energy and reserves, which are the same original physical product but distinct commercial products. Producers can sell both products in order to maximize their profits, subject to the units' capacities. From the market's point of view, the goal is to get the required quantities at the minimum possible cost. This requires allocating plants efficiently for the energy and reserve markets. ASM clearing mechanisms aim at dispatching the required AS at minimum cost. For the work presented in this paper, the results obtained by a linear programming approach and mixed integer non-linear programming in the case that complex bids are considered are presented. These two methods have been implemented in General Algebraic Modeling System (GAMS) [34].

The ancillary services dispatch by linear programming optimization can be formulated by (2)-(8) for Regulation Down, Regulation Up, Spin Reserves and Non-Spin Reserves. Regulation Down dispatch does not have an effect on the maximum available capacity of market players $[13,18,35,36]$.

Minimization of AS dispatch costs:

$\sum_{k=1}^{4} \sum_{i=1}^{N} \sum_{t=1}^{T}\left(P R_{k, i, t}+P E_{k, i, t}\right) \cdot X_{k, i, t}$
The minimization of the objective function presented in (2) is subjected to the following constraints:

The requirements must be achieved:

$\sum_{t=1}^{T} \sum_{k=1}^{4} \sum_{i=1}^{N} X_{k, i, t}=Q_{k, t}$

Total bid capacity limit:

$\sum_{t=1}^{T} \sum_{i=1}^{N} \sum_{k=2}^{4} X_{k, i, t} \leqslant C \max _{i, t} \quad t \in\{1, \ldots, T\} ; \quad \forall i \in\{1, \ldots, N\}$

Bid capacity limit for each service:

$0 \leqslant X_{k, i, t} \leqslant C_{k, i, t}$

where $N$ is the total number of bids; $i$ is the bid index $(i=1,2, \ldots$. $N$ ); $k$ is the ancillary service index ( 1 for Regulation Down, 2 for Regulation Up, 3 for spin, and 4 for non-spin); $T$ is the total number periods in hours; $t$ is the period index $(t=1,2, \ldots, T) ; Q_{k, t}$ is the total capacity requirement for ancillary service $k$ in period $t$; $C \max _{i, t}$ is the maximum capacity of bid $i$ in period $t ; C_{k, i, t}$ is the capacity of bid $i$ for ancillary service $k$ in period $t ; X_{k, i, t}$ is the accepted capacity of bid $i$ for ancillary service $k$ in period $t ; P R_{k, i, t}$ is the capacity reserve price of bid $i$ for ancillary service $k$ in period $t ; P E_{k, i, t}$ is the energy reserve price of bid $i$ for ancillary service $k$ in period $t$.

This paper considers complex bids that can accommodate the objectives of the producers and the needs of the system operator. In this context, three types of complex bids were considered. The mathematical problem considering the complex bids can be formulated by Eqs. (2)-(5) plus the following constraints:

Minimum remuneration for each bid:

$\sum_{k=1}^{4} \sum_{i=1}^{N} \sum_{t=1}^{T}\left(P R_{k, i, t}+P E_{k, i, t}\right) \cdot X_{k, i, t} \geqslant M m_{i}$

Minimum working hours for each bid:

$\begin{aligned} \sum_{t=1}^{T} Y_{(k, i, t)} & \geqslant Y l_{(k, i, t)}, \quad \forall t \in\{1, \ldots, T\} ; \quad \forall i \in\{1, \ldots, N\} \quad \forall k \\ & \in\{1,2,3,4\} ; \quad Y_{(k, i, t)} \in\{0,1\}\end{aligned}$ 


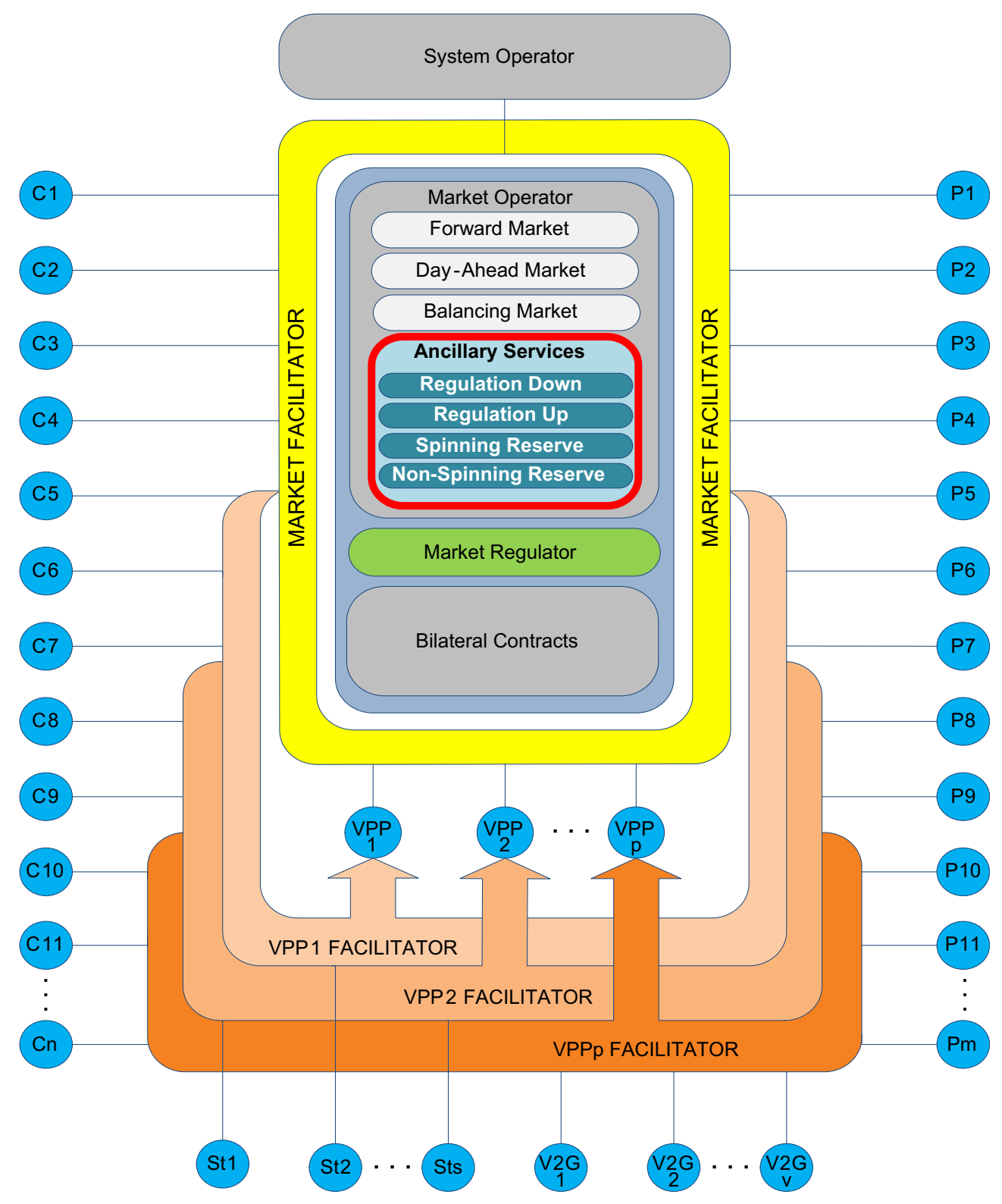

Fig. 2. MASCEM architecture (adapted from [37]).

Maximum working hours for each bid:

$\sum_{t=1}^{T} Y_{(k, i, t)} \leqslant \operatorname{Yup}_{(k, i, t)}, \quad \forall t \in\{1, \ldots, T\} ; \quad \forall i \in\{1, \ldots, N\} ; \quad \forall k$

$$
\in\{1,2,3,4\} ; \quad Y_{(k, i, t)} \in\{0,1\}
$$

Minimum consecutive working hours for each bid:

$$
\begin{aligned}
\sum_{t=2}^{T} Y_{(k, i, t)}-Y_{(k, i, t-1)} & \geqslant M h_{(i)}, \quad \forall t \in\{1, \ldots, T\} ; \quad \forall i \\
& \in\{1, \ldots, N\} ; \quad \forall k \in\{1,2,3,4\} ; \quad Y_{(k, i, t)} \\
& \in\{0,1\}
\end{aligned}
$$

Maximum consecutive working hours for each bid:

$$
\begin{aligned}
\sum_{t=2}^{T} Y_{(k, i, t)}-Y_{(k, i, t-1)} & \leqslant 24, \quad \forall t \in\{1, \ldots, T\} ; \quad \forall i \\
& \in\{1, \ldots, N\} ; \quad \forall k \in\{1,2,3,4\} ; \quad Y_{(k, i, t)} \\
& \in\{0,1\}
\end{aligned}
$$

where $M m_{i}$ is the minimum limit of remuneration for the whole $24 \mathrm{~h} ; Y_{k, i, t}$ is the decision variable of bid $i$ for service $k$ in period $t$; $Y l_{k, i, t}$ is the minimum working hours of bid $i$ for service $k$ in each time period; Yup $_{k, i, t}$ is the maximum working hours of bid $i$ for service $k$ in each time period; $M h_{i}$ is the minimum consecutive working hours of bid $i$.

Fig. 3 presents a block diagram of the proposed methodology to solve AS market clearing price, considering the market clearing mechanism based on linear programming (normal dispatch) and complex bids based on mixed integer non-linear programming (dispatch with complex bids).

The mathematical problem for the two dispatch models (normal and with complex bids) has been implemented using GAMS [34]. For the normal dispatch, taking into account the nature of the problem (linear programming) the CPLEX solver has been the chosen one and for the dispatch with complex bids (mixed integer non-linear programming) the solvers CPLEX and CONOPT have been chosen. 
Table 1

Input data for the forecast of each period and each service.

\begin{tabular}{|c|c|c|c|c|c|c|c|c|c|c|c|c|c|c|c|c|c|c|}
\hline \multirow{2}{*}{$\begin{array}{l}\text { Day } \\
\text { Historical data }\end{array}$} & \multicolumn{2}{|c|}{$n-8$} & \multicolumn{2}{|c|}{$n-7$} & \multicolumn{2}{|c|}{$n-6$} & \multicolumn{2}{|c|}{$n-5$} & \multicolumn{2}{|c|}{$n-4$} & \multicolumn{2}{|c|}{$n-3$} & \multicolumn{2}{|c|}{$n-2$} & \multicolumn{2}{|c|}{$n-1$} & \multicolumn{2}{|l|}{$N$} \\
\hline & FD & RS & FD & RS & FD & RS & FD & RS & FD & RS & FD & $\mathrm{RS}$ & FD & $\mathrm{RS}$ & FD & $\mathrm{RS}$ & FD & $\mathrm{RS}$ \\
\hline \multicolumn{19}{|l|}{ Hour } \\
\hline$h-3$ & - & - & - & - & - & - & - & - & - & - & - & - & - & - & - & - & $\times$ & $X$ \\
\hline$h-2$ & - & - & - & - & - & - & - & - & - & - & - & - & - & - & - & - & $\times$ & $X$ \\
\hline$h-1$ & - & - & - & $\times$ & - & - & - & - & - & - & - & - & - & - & - & $\times$ & $\times$ & $X$ \\
\hline$h$ & - & $\times$ & $\times$ & $\times$ & $\times$ & $\times$ & $\times$ & $\times$ & $x$ & $x$ & $\times$ & $\times$ & $\times$ & $\times$ & $\times$ & $\times$ & - & $\mathrm{F}$ \\
\hline$h+1$ & - & - & - & - & - & - & - & - & - & - & - & - & - & - & - & $\times$ & - & - \\
\hline
\end{tabular}

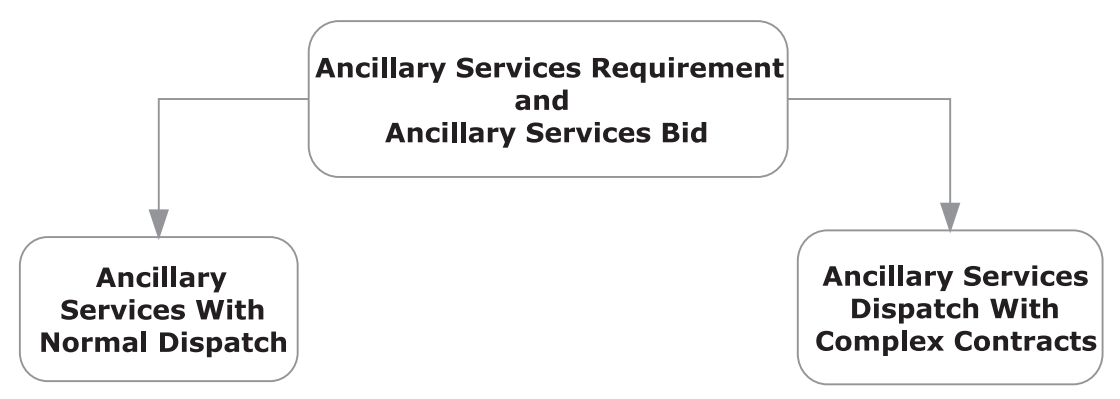

Fig. 3. Block diagram of the proposed methodology.

\section{Case study}

\subsection{CAISO market data}

The California Independent System Operator (CAISO) publishes every day the day-ahead hourly final ancillary services (AS) requirements, specifying the amount of energy to be guaranteed in each one of those AS. This information can be obtained online [38].

The present case-study considers the data obtained for the 2007 winter season, for four AS: Regulation Down (RD), Regulation Up (RU), Spin Reserve (SR), and Non-Spin Reserve (NSR). For all services, day-ahead hourly energy requirement and load data of 48 days before the day whose AS are to be forecasted are used to build the forecasting model. The last week of winter season, that corresponds to the last week of February (from February 19 till February 25), has been used to test the performance of the proposed Artificial Neural Network (ANN). The hourly data used in the present study correspond to the period from January 2 till February $18,2007$.

Ref. [32] clearly shows that SR requirements are highly related to the Forecasted Demand (FD) and therefore FD is used as an exogenous variable to predict SR requirements of the CAISO controlled grid. Our forecasting methodology considers that this correlation can be applied to other AS and the results are analyzed.

The historical data collected in CAISO website include values of load and AS to the 48 days before the test week plus the test week values, corresponding to a total of 1320 periods $(=24 * 48+24 * 7=$ $1152+168)$, for each AS. The data of the previous $192 \mathrm{~h}$ is needed to predict the AS requirements for hour $h$; therefore, 960 ( $=1152-$ 192 ) input vectors are used to train the network.

Fifteen ancillary services bids that belong to a case study developed by the authors [39] are used in the present paper.

\subsection{AS requirements}

Fig. 4 presents the AS requirements obtained for each service, $R D$ in $4 a, R U$ in $4 b, S R$ in $4 c$, and NSR in $4 d$, and are obtained by three different sources: the proposed ANN used forecast, the forecast performed by CAISO, and the current values.
Table 2 shows the Mean Absolute Percentage Error (MAPE) values for the results obtained for AS requirements. Regarding the AS requirement forecast results, MAPE values are obtained comparing the values of the forecasts obtained by CAISO and with the proposed ANN method, with the current value of each AS requirement. The only AS for which CAISO provides a MAPE value better than the proposed ANN approach is the RU service. Among all services, NSR is the AS with the worse MAPE results for both forecast methodologies.

\subsection{AS clearing}

This subsection presents the application of linear programming (LP) and mixed integer non-linear programming (MINLP) approaches in order to determine the ancillary services (AS) reserve cost, AS energy cost as well as the market clearing price to the case study. The LP and MINLP approaches are for the normal dispatch and dispatch with complex bids respectively.

The input data for the two presented approaches can be found in Ref. [39]. These data correspond to $24 \mathrm{~h}$ and include the AS bids for a set of 15 players expressed in MW, the values of the required power reserve for each service in MW, the reserve prices in m.u./ MW and energy prices in m.u./MW h, where m.u. stands for monetary units. Table 3 shows an example of these input data for hour 1. The input data of complex bids are presented in Table 4.

Fig. 5a presents the reserve cost, in monetary units (m.u.), for a period of $24 \mathrm{~h}$, obtained for the dispatch with complex bids and for the normal dispatch, respectively. One can see that normal dispatch results and dispatch with complex bids results are very close, with differences only in hours $13,16,17$, and 19 . These differences never exceed $4 \%$ except in hour 16 where the difference is $4.44 \%$. This is in evidence in Fig. 5b, which presents the difference between the two approaches results.

The weight relation of the energy costs in comparison with the reserve costs is very high. The reserve costs depend on the resulting MCP. This means that the reserve cost solutions will be the total required energy multiplied by the MCP. Higher values of MCP in dispatch for a given service with the energy costs parcel presents higher difference, which weight is higher in comparison with the reserve costs. 
(a) Regulation Down

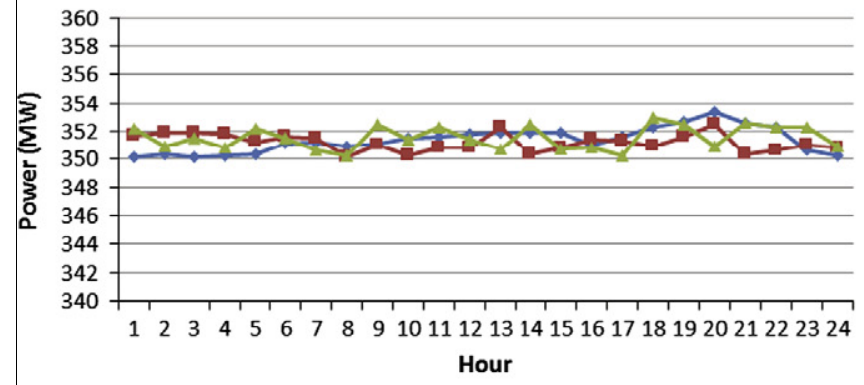

(c) Spinning Reserve

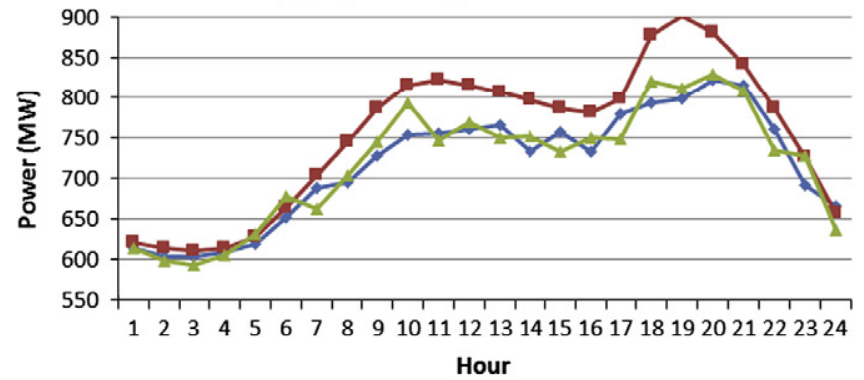

(b) Regulation Up

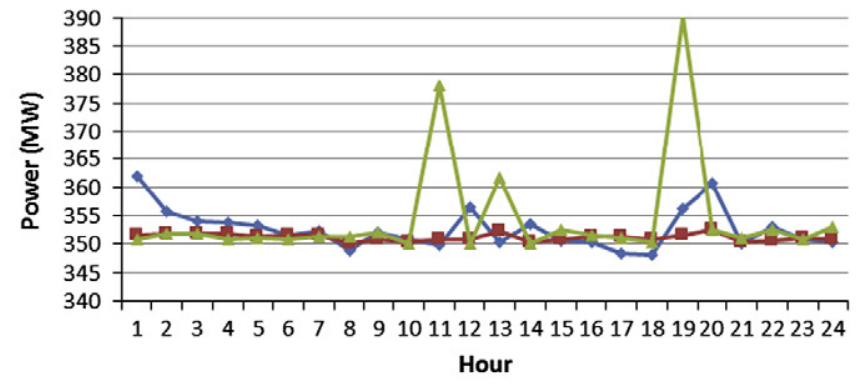

(d) Non-Spinning Reserve

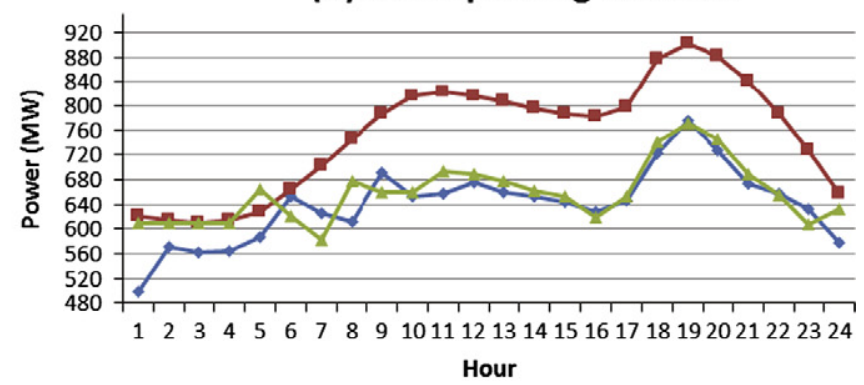

Fig. 4. AS requirements for each period.

Table 2

MAPE values for ancillary services requirements.

\begin{tabular}{llr}
\hline RD (\%) & ANN & 0.253 \\
& Caiso & 0.294 \\
$\mathrm{RU}(\%)$ & ANN & 1.725 \\
& Caiso & 0.990 \\
$\mathrm{SR}(\%)$ & ANN & 2.405 \\
& Caiso & 4.800 \\
NSR (\%) & ANN & 4.710 \\
& Caiso & 14.737 \\
\hline
\end{tabular}

The energy costs, in monetary units (m.u.) for $24 \mathrm{~h}$, obtained for the dispatch with complex bids and for the normal dispatch are depicted in Fig. 6a. As shown in Fig. 6b the differences between the normal dispatch and dispatch with complex bids are small, never exceeding $2.5 \%$.

Fig. 7a depicts the final cost for $24 \mathrm{~h}$ for the dispatch with complex bids and for the normal dispatch, which is the reserve cost plus the energy cost. Fig. 7b shows the difference in percentage between the dispatch with complex bids and the normal dispatch.

Fig. 8a-d presents the MCP for the $24 \mathrm{~h}$ considering the normal dispatch and the dispatch with complex bids.

The MINLP and LP approaches have been tested on a PC compatible with two processors Intel Xeon X5450 3.0 GHz, each one with 4 Cores, 4 GB of Random-Access-Memory (RAM) and Windows Server 2008 Operating System. LP spends around $0.2 \mathrm{~s}$ while MINLP spends around $1.7 \mathrm{~s}$ of computational time for the presented case study.

Table 3

Input data for AS market clearing price.

\begin{tabular}{|c|c|c|c|c|c|c|c|c|c|c|c|c|c|}
\hline \multirow[t]{2}{*}{$\begin{array}{l}\text { Hour } 1 \\
\text { BID }\end{array}$} & \multicolumn{3}{|c|}{ Regulation Down } & \multicolumn{3}{|c|}{ Regulation Up } & \multicolumn{3}{|c|}{ Spin Reserve } & \multicolumn{3}{|c|}{ Non-Spin Reserve } & \multirow{2}{*}{$\begin{array}{l}\text { Max. } \\
\text { power } \\
(\mathrm{MW})\end{array}$} \\
\hline & $\begin{array}{l}\text { Quantity } \\
\text { (MW) }\end{array}$ & $\begin{array}{l}\text { PR (m.u./ } \\
\text { MW) }\end{array}$ & $\begin{array}{l}\text { PE } \\
\text { (m.u./ } \\
\text { MW h) }\end{array}$ & $\begin{array}{l}\text { Quantity } \\
\text { (MW) }\end{array}$ & $\begin{array}{l}\text { PR (m.u./ } \\
\text { MW) }\end{array}$ & $\begin{array}{l}\text { PE } \\
\text { (m.u./ } \\
\text { MW h) }\end{array}$ & $\begin{array}{l}\text { Quantity } \\
\text { (MW) }\end{array}$ & $\begin{array}{l}\text { PR (m.u./ } \\
\text { MW) }\end{array}$ & $\begin{array}{l}\text { PE } \\
\text { (m.u./ } \\
\text { MW h) }\end{array}$ & $\begin{array}{l}\text { Quantity } \\
\text { (MW) }\end{array}$ & $\begin{array}{l}\text { PR (m.u./ } \\
\text { MW) }\end{array}$ & $\begin{array}{l}\text { PE } \\
\text { (m.u./ } \\
\text { MW h) }\end{array}$ & \\
\hline 1 & 50 & 5 & 30 & 48 & 3 & 36 & 55 & 2 & 34 & 10 & 6 & 25 & 113 \\
\hline 2 & 20 & 6 & 34 & 37 & 7 & 24 & 85 & 8 & 22 & 15 & 6 & 28 & 137 \\
\hline 3 & 50 & 4 & 38 & 45 & 6 & 26 & 50 & 3 & 33 & 20 & 2 & 49 & 115 \\
\hline 4 & 15 & 4 & 40 & 48 & 4 & 36 & 10 & 7 & 30 & 20 & 3 & 31 & 78 \\
\hline 5 & 36 & 8 & 24 & 11 & 4 & 37 & 50 & 4 & 37 & 65 & 4 & 50 & 126 \\
\hline 6 & 90 & 6 & 35 & 38 & 6 & 27 & 50 & 7 & 28 & 85 & 2 & 39 & 173 \\
\hline 7 & 44 & 7 & 25 & 39 & 6 & 22 & 90 & 7 & 24 & 10 & 4 & 45 & 139 \\
\hline 8 & 45 & 5 & 32 & 41 & 6 & 25 & 35 & 8 & 27 & 30 & 4 & 39 & 106 \\
\hline 9 & 20 & 4 & 40 & 11 & 2 & 34 & 60 & 3 & 32 & 60 & 8 & 26 & 131 \\
\hline 10 & 10 & 7 & 28 & 46 & 4 & 48 & 95 & 8 & 24 & 20 & 5 & 39 & 161 \\
\hline 11 & 20 & 4 & 46 & 47 & 6 & 22 & 35 & 5 & 42 & 20 & 4 & 46 & 102 \\
\hline 12 & 15 & 4 & 39 & 15 & 4 & 41 & 70 & 3 & 47 & 60 & 6 & 25 & 145 \\
\hline 13 & 10 & 5 & 30 & 48 & 6 & 26 & 10 & 7 & 25 & 90 & 6 & 26 & 148 \\
\hline 14 & 5 & 6 & 27 & 34 & 2 & 38 & 65 & 7 & 24 & 50 & 2 & 48 & 149 \\
\hline 15 & 10 & 4 & 35 & 12 & 7 & 28 & 20 & 5 & 49 & 20 & 8 & 28 & 52 \\
\hline Required & & 350.1543 & & & 362.0124 & & & 613.3876 & & & 499.2676 & & 1824.8218 \\
\hline
\end{tabular}


Table 4

Input data of complex bids.

\begin{tabular}{|c|c|c|c|c|c|c|c|c|c|c|c|c|c|}
\hline \multirow[t]{2}{*}{ Bids } & \multirow[t]{2}{*}{ Minimum obtained money (m.u.) } & \multicolumn{4}{|c|}{ Minimum working hours } & \multicolumn{4}{|c|}{ Maximum working hours } & \multicolumn{4}{|c|}{ Minimum consecutive working hours } \\
\hline & & $\mathrm{RD}$ & RU & SR & NSR & $\mathrm{RD}$ & RU & SR & NSR & $\mathrm{RD}$ & RU & SR & NSR \\
\hline 1 & 60,000 & 3 & 2 & 2 & 1 & 11 & 13 & 11 & 11 & 1 & 1 & 1 & 1 \\
\hline 2 & 60,000 & 0 & 3 & 1 & 1 & 10 & 12 & 12 & 11 & 2 & 2 & 2 & 2 \\
\hline 3 & 65,000 & 2 & 4 & 1 & 2 & 11 & 11 & 11 & 13 & 1 & 1 & 1 & 1 \\
\hline 4 & 50,000 & 4 & 0 & 1 & 4 & 9 & 14 & 11 & 14 & 1 & 1 & 1 & 1 \\
\hline 5 & 55,000 & 0 & 0 & 1 & 2 & 13 & 11 & 14 & 11 & 1 & 1 & 1 & 1 \\
\hline 6 & 30,000 & 2 & 2 & 5 & 3 & 10 & 11 & 12 & 12 & 0 & 0 & 0 & 0 \\
\hline 7 & 60,000 & 0 & 1 & 3 & 1 & 11 & 10 & 15 & 12 & 1 & 1 & 1 & 1 \\
\hline 8 & 72,000 & 2 & 1 & 5 & 0 & 13 & 11 & 10 & 11 & 2 & 2 & 2 & 2 \\
\hline 9 & 35,000 & 0 & 2 & 0 & 3 & 12 & 13 & 11 & 12 & 3 & 3 & 3 & 3 \\
\hline 10 & 25,000 & 1 & 4 & 2 & 4 & 12 & 11 & 12 & 14 & 1 & 1 & 1 & 1 \\
\hline 11 & 50,000 & 3 & 2 & 1 & 0 & 14 & 14 & 12 & 10 & 0 & 0 & 0 & 0 \\
\hline 12 & 60,000 & 0 & 1 & 0 & 1 & 11 & 10 & 11 & 11 & 0 & 0 & 0 & 0 \\
\hline 13 & 45,000 & 1 & 0 & 3 & 2 & 9 & 8 & 13 & 14 & 2 & 2 & 2 & 2 \\
\hline 14 & 55,000 & 1 & 0 & 2 & 2 & 10 & 11 & 13 & 11 & 3 & 3 & 3 & 3 \\
\hline 15 & 45,000 & 4 & 1 & 3 & 1 & 11 & 13 & 12 & 11 & 1 & 1 & 1 & 1 \\
\hline
\end{tabular}

(a) Reserve cost

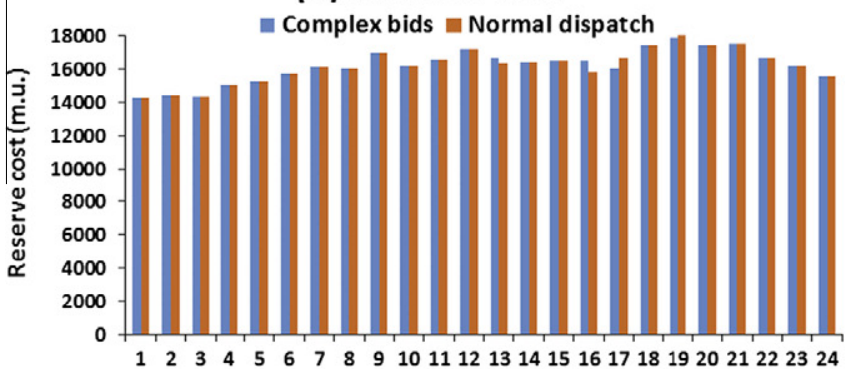

Hour (b) Difference

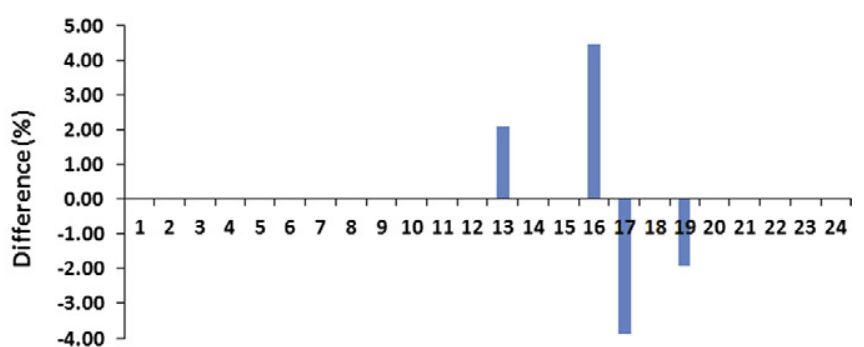

Hour

Fig. 5. Reserve cost.

(a) Energy cost

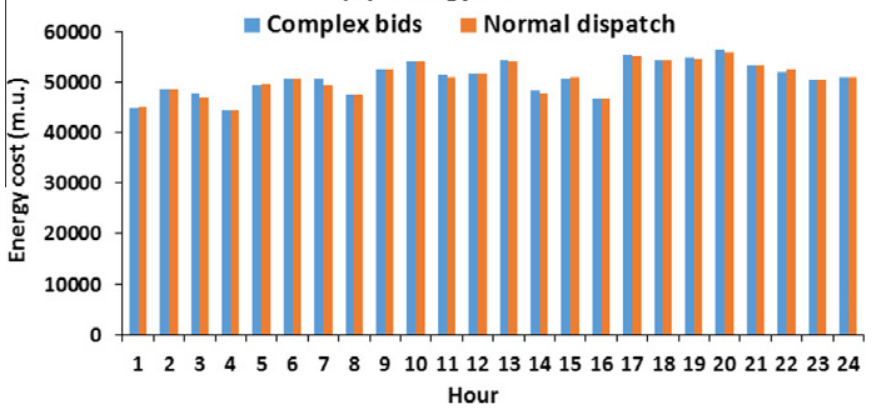

(b) Difference

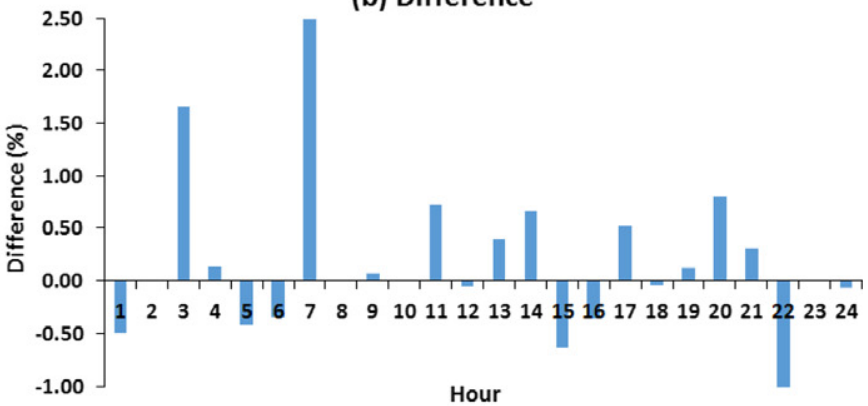

Fig. 6. Energy cost. (a) Final cost

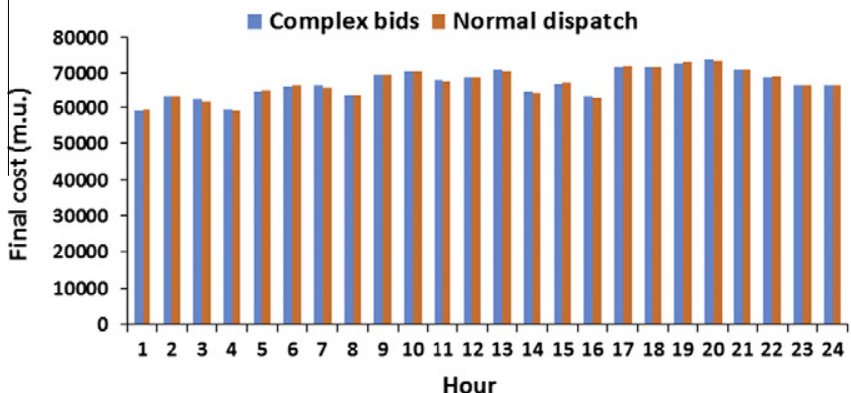

(b) Difference

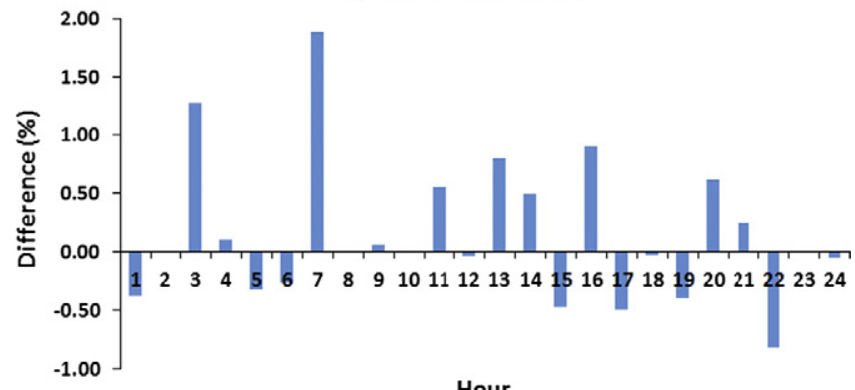

Hour

Fig. 7. Total cost. 
(a) Regulation Down
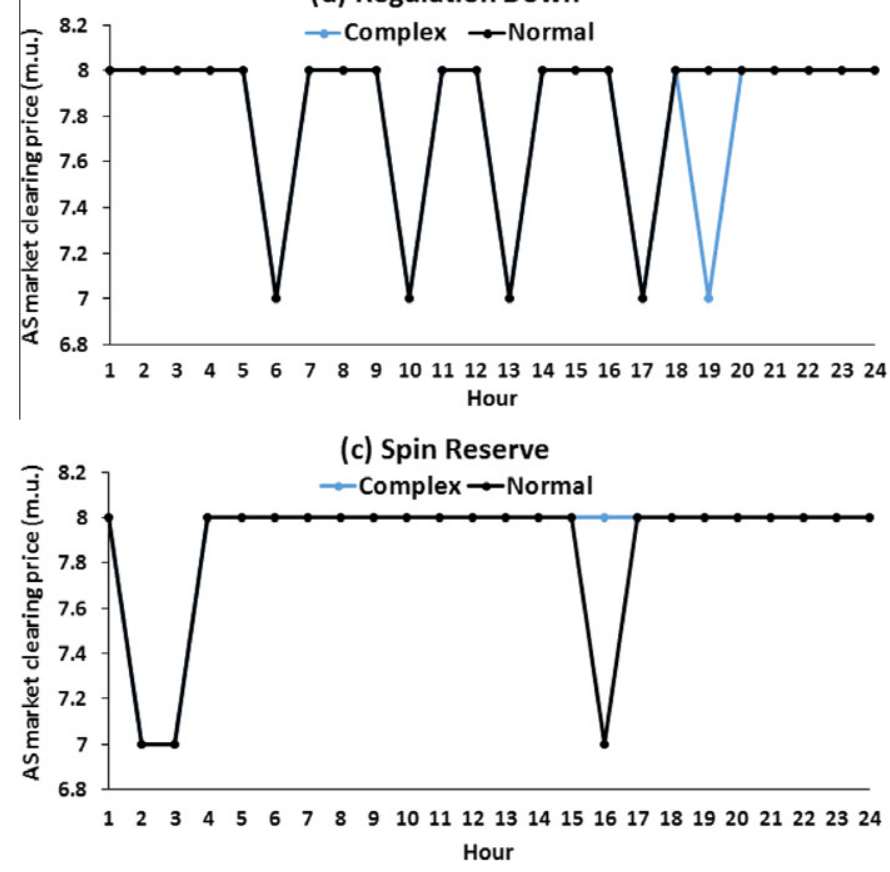

(b) Regulation Up

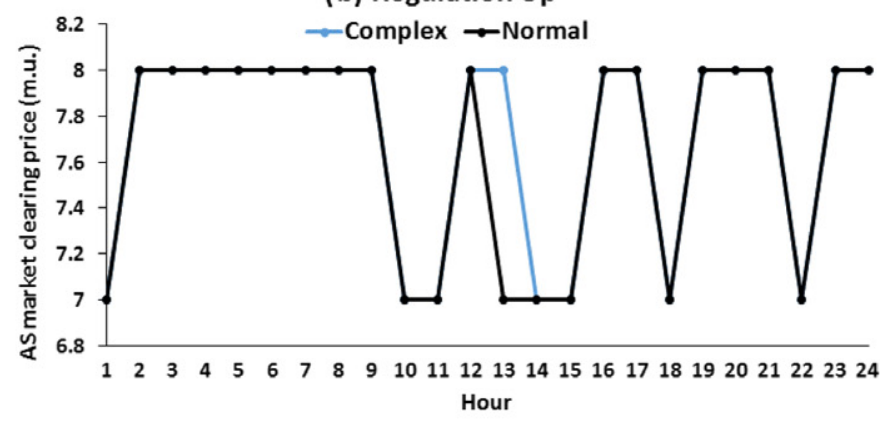

(d) Non-Spin Reserve

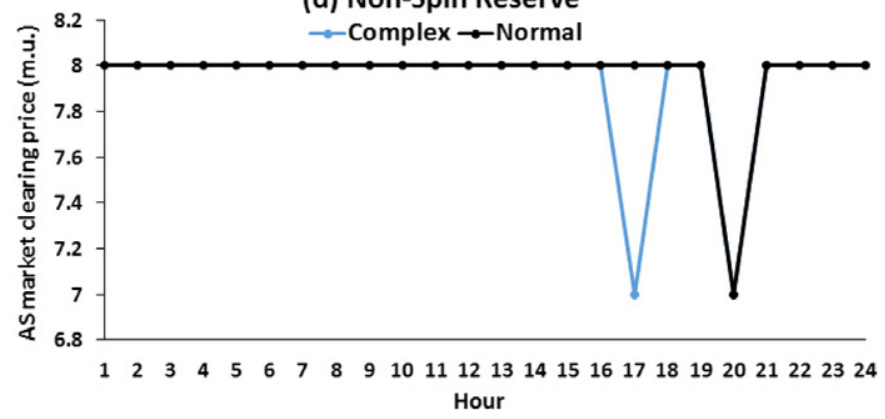

Fig. 8. Market clearing price.

\section{Conclusions}

Providing adequate decision support for electricity market players requires the use of decision support tools which can be effectively based on simulation studies. In order to make decision support effective, all business opportunities must be considered. Ancillary services are an important component of electricity markets as they represent a business opportunity for market players. Therefore they must be included in electricity market simulation, so that it is possible to support market participants' decisions.

This paper proposes a deterministic approach to solve a bid-based ancillary services dispatch in an electricity market and use an Artificial Neural Network approach for day-ahead prediction of Regulation Down, regulation-up, Spin Reserve and Non-Spin Reserve requirements.

A case study, with real data from the California Independent System Operator, allows comparing the results obtained with the method without complex bids which is based on linear programming with those obtained with the method considering complex bids based on mixed integer non-linear programming. Electricity market operation shows the importance of using adequate methodologies for ancillary services dispatch in a market environment.

The inclusion of ancillary service markets in an electricity market simulator, like MASCEM, allows electricity market players to make simulation studies, considering a combined participation in several markets, including energy and ancillary services markets, allowing them to define adequate bidding strategies.

\section{Acknowledgements}

This work is supported by FEDER Funds through COMPETE program and by National Funds through FCT under the projects FCOMP-01-0124-FEDER: PEst-OE/EEI/UI0760/2011, PTDC/EEAEEL/099832/2008, PTDC/SEN-ENR/099844/2008, PTDC/SEN-ENR/ 122174/2010, and PTDC/EEA-EEL/122988/2010.

\section{References}

[1] Li G, Shi J. Agent-based modeling for trading wind power with uncertainty in the day-ahead wholesale electricity markets of single-sided auctions. Appl Energy 2012;99:13-22.
[2] Joung M, Kim J. Assessing demand response and smart metering impacts on long-term electricity market prices and system reliability. Appl Energy 2013;101:441-8

[3] Kumar A, Gao WZ. Pattern of secure bilateral transactions ensuring power economic dispatch in hybrid electricity markets. Appl Energy 2009;86: 1000-10.

[4] Ghadikolaei HM, Tajik E, Aghaei J, Charwand M. Integrated day-ahead and hour-ahead operation model of discos in retail electricity markets considering DGs and $\mathrm{CO}_{2}$ emission penalty cost. Appl Energy 2012;95:174-85.

[5] Ghazvini MAF, Canizes B, Vale Z, Morais H. Stochastic short-term maintenance scheduling of GENCOs in an oligopolistic electricity market. Appl Energy 2013;101:667-77.

[6] Fragaki A, Andersen AN. Conditions for aggregation of CHP plants in the UK electricity market and exploration of plant size. Appl Energy 2011;88: 3930-40.

[7] Pandzic H, Kuzle I, Capuder T. Virtual power plant mid-term dispatch optimization. Appl Energy 2013;101:134-41.

[8] Kargarian A, Raoofat M, Mohammadi M. Reactive power market management considering voltage control area reserve and system security. Appl Energy 2011:3832-40.

[9] Shahidehpour M, Yamin H, Li Z. Market operations in electric power systems: forecasting, scheduling, and risk management. New York: Institute of Electrical and Electronics Engineers, Wiley-Interscience; 2002.

[10] Aghaei J, Shayanfar HA, Amjady N. Joint market clearing in a stochastic framework considering power system security. Appl Energy 2009;86: $1675-82$.

[11] Rabiee A, Shayanfar H, Amjady N. Multiobjective clearing of reactive power market in deregulated power systems. Appl Energy 2009;86:1555-64.

[12] Hooshmand RA, Parastegari M, Morshed MJ. Emission, reserve and economic load dispatch problem with non-smooth and non-convex cost functions using the hybrid bacterial foraging-Nelder-Mead algorithm. Appl Energy 2012;89:443-53.

[13] Vale ZA, Ramos C, Faria P, Soares JP, Canizes BR, Khodr HM. Ancillary service market simulation. In: IEEE T\&D Asia 2009, Seoul, Korea; 2009.

[14] Ostergaard PA. Ancillary services and the integration of substantial quantities of wind power. Appl Energy 2006;83:451-63.

[15] Amjady N, Keynia F. A new spinning reserve requirement forecast method for deregulated electricity markets. Appl Energy 2010;87:1870-9.

[16] Willis L, Finney J, Ramon G. Computing the cost of unbundled services. IEEE Comput Appl Power 1996;9:16-21.

[17] FERC FERC-. Transmission open access. Promoting wholesale competition through open access non-discriminatory transmission services by public utilities; recovery of stranded costs by public utilities and transmitting utilities; 1996.

[18] Vale ZA, Ramos C, Faria P, Soares JP, Canizes B, Khodr HM. Ancillary services dispatch using linear programming and genetic algorithm approaches. MELECON 2010-2010. In: 15th IEEE mediterranean electrotechnical conference; 2010. p. 667-72.

[19] Faria P, Vale Z, Soares J, Khodr H, Canizes B. ANN based day-ahead ancillary services forecast for electricity market simulation. In: The 15th IEEE mediterranean electrotechnical conference (MELECON 2010), La Valletta, Malta; 2010. 
[20] Cheung KW, Shamsollahi P, Sun D, Milligan J, Potishnak M. Energy and ancillary service dispatch for the interim ISO New England electricity market. In: Proceedings of the 21st international conference on power industry computer applications; 1999. p. 47-53.

[21] Wolak F, Nordhaus R, Shapiro C. Preliminary report on the operating ancillary services markets of California independent system operator. In: ISO C, editor; 1998.

[22] Oren S. Design of ancillary service market. In: 34th Hawaii international conference on system sciences, Hawaii; 2001. p. 9.

[23] Zammit MAB, Hill DJ, Kaye RJ. Designing ancillary services markets for power system security. IEEE Trans Power Syst 2000;15:675-80.

[24] Wu T, Rothleder M, Alaywan Z, Papalexopoulos AD. Pricing energy and ancillary services in integrated market systems by an optimal power flow. IEEE Trans Power Syst 2004;19:339-47.

[25] Wang JH, Shahidehpour M, Li ZY. Contingency-constrained reserve requirements in joint energy and ancillary services auction. IEEE Trans Power Syst 2009;24:1457-68.

[26] Vale Z, Pinto T, Praca I, Morais H. MASCEM: electricity markets simulation with strategic agents. IEEE Intell Syst 2011;26:9-17.

[27] CAISO. Market optimization details. In: ISO C, editor; 2009.

[28] CIGRÉ. Methods and tools for costing ancillary services; 2001.

[29] Vale ZA, Morais H, Cardoso M, Ramos C. Distributed generation producers' reserve management. IEEE Power Energy Soc Gen Meet 2008;1-11:2367-73.

[30] Faria P, Vale Z, Soares J, Khodr H. ANN based day-ahead spinning reserve forecast for electricity market simulation. In: The 15th international conference on intelligent system applications to power systems Curitiba, Brazil; 2009.

[31] Chang HH, Yang HT. Applying a non-intrusive energy-management system to economic dispatch for a cogeneration system and power utility. Appl Energy 2009;86:2335-43.

[32] Pindoriya NA, Singh N, Singh SK. Forecasting the day-ahead spinning reserve requirement in competitive electricity market. IEEE Power Energy Soc Gen Meet 2008;1-11:3697-704.

[33] Kecman V. Learning and soft computing: support vector machines, neural networks, and fuzzy logic models. Cambridge: MIT Press; 2001.

[34] Sorg A, Wellssow WH, Lietz T, Sievers D, Zdrallek M. The impact of distribution curve types of component indices in electric power system reliability evaluation. Saf Reliab 1999;1 \& 2:97-102.

[35] Papalexopoulos A, Singh H. On the various design options for ancillary services markets. In: 34th Hawaii international conference on system sciences, Hawaii; 2001.

[36] Pereira AJC, Vale ZA, Moura AMe, Pinto JAD. Provision and costs of ancillary services in a restructured electricity market. In: International conference on renewable energy and power quality, Barcelona; 2004.

[37] Santos G, Pinto T, Morais H, Vale Z, Praca I. Multi-agent simulation of continental, regional, and micro electricity markets. Database and Expert Systems Applications (DEXA), 2012. In: 23rd international workshop; 2012. p. 331-335.

[38] http://www.caiso.com. [accessed 02.13].

[39] http://gecad.isep.ipp.pt:8585/case_studies.php [accessed 02.13]. 